\section{South Asian diabetic macular oedema treated with ranibizumab (ADMOR)—real-life experience}

F Ghanchi and CA Hazel

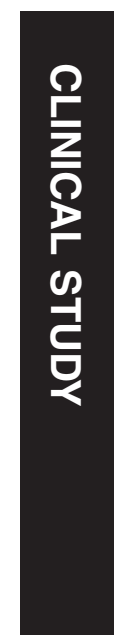

\begin{abstract}
Purpose Diabetic macular oedema (DMO) is a leading cause for visual impairment in the working age population in the UK.

Ranibizumab has been shown to be effective in treatment of DMO in studies based on mainly Caucasian populations. This study reports the 12-month outcome in a cohort of South Asian subjects with DMO treated with ranibizumab. Methods DMO in 51 eyes of 41 South Asian patients was treated with ranibizumab $0.5 \mathrm{mg}$ according to the modified DRCRnet protocol I. Visual acuity (VA) and central macular thickness (CMT) were recorded at baseline, 3, 6, and 12 months. Results were compared for eyes with different baseline visual acuities and different baseline macular thicknesses. Results Over the 12-month period, the mean ETDRS VA increased from $55.3 \pm 13.4$ letters to $63.8 \pm 15.2$ letters for all eyes. At 12 months, $70.6 \%$ eyes gained 5 or more letters acuity and

occurring in $8.9 \%$ of type 1 diabetics and $4.7 \%$ of type $2 .{ }^{1}$ Several multi-centre randomised trials have demonstrated the efficacy of ranibizumab for DMO. ${ }^{2-6}$ Ranibizumab has been introduced as a treatment option within the NHS, for DMO where central retinal thickness exceeds $400 \mu \mathrm{m}$ (http://guidance.nice.org.uk/TA274/

Guidance/pdf/English).

The prevalence of diabetes as well as the severity of diabetic retinopathy is higher in South Asians compared with Caucasians. ${ }^{7-11}$ Furthermore, diabetics of South Asian origin are twice as likely to develop DMO as Caucasians. ${ }^{1}$ However, the pivotal studies on ranibizumab use in DMO were primarily based on Caucasian populations, resulting in little useful data on South Asians. This paper describes the first cohort of South Asian patients with DMO who underwent ranibizumab treatment for DMO and completed 12 months follow-up.
\end{abstract} $17.6 \%$ eyes gained 15 letters or more. During the same period, the mean CMT decreased from $532 \pm 129$ to $318 \pm 136 \mu \mathrm{m}$. Eyes that had received previous laser treatments had a mean letter gain of 9.2 letters, compared with 8.5 for all eyes at 12 months.

Conclusions Ranibizumab $0.5 \mathrm{mg}$ is safe and effective at reversing vision loss due to DMO in patients of South Asian origin at 12 months. Ranibizumab treatment appears to be effective in patients with longstanding DMO who received prior laser treatments. Further studies are needed to define the longterm outcome in patients of different ethnicity and DMO.

Eye (2016) 30, 133-138; doi:10.1038/eye.2015.209; published online 23 October 2015

\section{Introduction}

Diabetic macular oedema (DMO) is a significant sight-threatening complication of diabetes,

\section{Materials and methods}

From the Macula Service database, all consecutive South Asian patients who had received an initial intravitreal ranibizumab $0.5 \mathrm{mg}$ treatment from February 2013 to November 2013, with a minimum of 12 months follow-up were selected. The real-life data were captured on electronic patient records (Medisoft Ltd, Leeds, UK) and digital retinal images with reports were assessed from the image database. All South Asian patients meeting NICE guidelines for DMO treatment were eligible for this study. The exclusion criteria followed Ranibizumab smpc. Thus, all consecutive South Asian patients receiving ranibizumab treatment for DMO were included in the study.

Each patient had a systematic assessment for both eyes including ETDRS visual acuity (VA) letter score, SD-OCT scan, and retinal examination at each visit scheduled 4 weeks
Bradford Teaching Hospitals NHS Foundation Trust, Bradford, UK

Correspondence: F Ghanchi, Ophthalmology Department, Bradford Teaching Hospitals NHS Foundation Trust, Duckworth Lane, Bradford BD9 6RJ, UK Fax: +44 (0)1274382390 E-mail: faruque.ghanchi@ bthft.nhs.uk

Received: 12 May 2015 Accepted in revised form: 3 September 2015 Published online: 23 October 2015
Tel: +44 (0)12 74382386 ; 
followed during clinical care of patients and data extraction for this research.

spectacles if worn habitually. Baseline was defined as the date on which the first ranibizumab injection was given. The 3-, 6-, and 12-month appointments were all within 3 weeks of 3,6, and 12 months post baseline, respectively. At each visit, the central macular thickness (CMT) was also recorded for both eyes using a spectral domain OCT (Ophthalmic Technologies Inc. (OTI), Toronto, ON, Canada).

A modified DRCRnet Protocol $\mathrm{I}^{12}$ was used under 'intention to treat' for all patients in this cohort. Patients with DMO received initial monthly injections of ranibizumab $0.5 \mathrm{mg}$ for 3 months. After this period, further monthly injections were given until 'success' or there was no further improvement $(<10 \%$ reduction in CMT or $<5$ letters vision gain) or the investigator considered treatment failure. (The 'success' criteria-VA reached 85 letters or CMT was $<250 \mu \mathrm{m})$. At each visit, the clinical and OCT data were entered prospectively on electronic patient records. Over the period of the study, the decisions to treat/re-treat were carried out by five Consultant Ophthalmologists, three Retina Specialists, and two Optometrist Specialists. All were well versed with the treatment protocol and subsequently, all data were verified by the lead Optometrist $(\mathrm{CH})$. Data were collected on the history of previous treatment for DMO including Bevacizumab, Triamcinolone, and laser, including timing of such treatment(s). Only one eye of one patient received additional macular laser treatment for DMO during the study period. One eye received preplanned PRP laser soon after the first injection.

\section{Statement of ethics}

All applicable institutional and governmental regulations concerning the ethical use of human volunteers were

\section{Results}

There were 41 patients ( 24 males and 17 females), with an average age of 63 years (range: $42-88$ years) at baseline. The mean $\mathrm{HbA} 1 \mathrm{c}$ at baseline was $69 \mathrm{mmol} / \mathrm{mol}$ (range 45-104). Of the 41 patients, 10 (5 males, 5 females) needed bilateral treatment, while 31 patients ( 19 males, 12 females) were treated in one eye only. The data for all eyes have been pooled to include 51 eyes in total.

Nine eyes were treatment-naïve (ie, no previous macular laser, intravitreal Triamcinolone injections, or pan-retinal photocoagulation). Thirty-four eyes had history of prior macular laser (median $=1.0$; range $=1-4$ ) and 20 eyes had received pan-retinal photocoagulation on at least one occasion. One eye had prior intravitreal bevacizumab injection and seven eyes had triamcinolone injections. The mean interval from any macular oedema treatment to initiation of ranibizumab treatment was 29 months (range 2-50 months, median 32 months). As there is lead in time for laser treatment in routine clinical practice, these patients would be considered to have chronic DMO.

The mean ETDRS VA for all eyes at baseline was $55.3 \pm 13.4$ letters (mean $\pm \mathrm{SD}$ ). This increased to $60.0 \pm 12.8$ letters, $62.3 \pm 14.0$ letters, and $63.8 \pm 15.2$ at 3,6 , and 12 months respectively (Table 1). During this study period, the mean number of injections was $7 \pm 2$ (range 3-11). The mean $( \pm S D)$ number of visits per subject over the 12 month period was $10 \pm 2$ with very good compliance.

Table 1 shows the number and percentage of patients who gained or lost 5, 10, or 15 letters over the 12 months. At 12 months, most patients ( 36 out or 51 eyes) had gained $\geq 5$ letters. Table 1 also shows reduction in the average CMT over the first 6 months, and subsequent stabilisation

Table 1 Mean \pm SD values for visual acuity (VA) and central macular thickness (CMT) shown for baseline, 3, 6, and 12 months

\begin{tabular}{|c|c|c|c|c|}
\hline & Baseline & 3 months & 6 months & 12 months \\
\hline \multirow{3}{*}{$\begin{array}{l}\text { BCVA (ETDRS letters } \pm \text { SD) } \\
\text { CMT }(\mu \mathrm{m} \pm \mathrm{SD})\end{array}$} & \multirow{3}{*}{$\begin{array}{c}55.3 \pm 13.4 \\
532 \pm 129\end{array}$} & $60.0 \pm 12.8$ & $62.3 \pm 14.0$ & $63.8 \pm 15.2$ \\
\hline & & $369 \pm 145$ & $318 \pm 136$ & $340 \pm 155$ \\
\hline & & 0-3 months & 0-6 months & 0-12 months \\
\hline Mean change in BCVA (ETDRS letters $\pm S D$ ) & & $4.7 \pm 8.4$ & $5.8 \pm 10.3$ & $8.5 \pm 10.0$ \\
\hline Mean change in CMT $(\mu \mathrm{m} \pm \mathrm{SD})$ & & $164 \pm 140$ & $214 \pm 161$ & $199 \pm 184$ \\
\hline \multicolumn{5}{|l|}{ Categorised BCVA outcome c.f. baseline } \\
\hline Gain of $\geq 5$ letters $n(\%)$ & & $24(47.1)$ & $30(58.8)$ & $36(70.6)$ \\
\hline Gain of $\geq 10$ letters $n(\%)$ & & $14(27.5)$ & $19(37.3)$ & $22(43.1)$ \\
\hline Gain of $\geq 15$ letters $n(\%)$ & & 7 (13.7) & $11(21.6)$ & $9(17.6)$ \\
\hline Loss of $\geq 5$ letters $n(\%)$ & & 7 (13.7) & $7(13.7)$ & $4(13.7)$ \\
\hline Loss of $\geq 10$ letters $n(\%)$ & & $1(2.0)$ & $3(5.9)$ & $1(2.0)$ \\
\hline Loss of $\geq 15$ letters $n(\%)$ & & $0(0.0)$ & $2(3.9)$ & $1(2.0)$ \\
\hline
\end{tabular}

Mean change in values between baseline and 12 months also shown for VA and CMT. 
between 6 and 12 months. There was a mean decrease in macular thickness of $199 \mu \mathrm{m}$ and a mean improvement of 8.5 letters between baseline and 12 months (Table 1). One patient lost more than 15 letters, who had poorly controlled diabetes throughout the study and was diagnosed with ischaemic maculopathy at 12 months (Table 1).

Table 2 shows the percentages of eyes (treatment-naïve and those with prior treatment) whose vision changed either 5,10 , or 15 letters over the 12-month period.

The mean baseline VA for the treatment-naïve eyes was $59.0 \pm 10.5$ letters (range: $42-73$ ), which increased to $64.2 \pm 10.4$ letters (range: $45-78$ ) at 12 months. The mean baseline CMT of this group was $519 \pm 131 \mu \mathrm{m}$, which reduced to $331 \pm 107$ at 12 months. The 42 eyes with prior treatment for diabetic retinopathy had a mean baseline VA of $54.5 \pm 13.9$ letters (range: $29-82$ ), which increased to $63.7 \pm 16.2$ letters (range: $16-85$ ) at 12 months. The mean baseline CMT of this group was $535 \pm 130 \mu \mathrm{m}$, which reduced to $338 \pm 167 \mu \mathrm{m}$ at 12 months (Table 2 ).

Table 2 The effect of treatment on VA for the $n=9$ eyes that were naïve to any prior treatment at baseline

\begin{tabular}{lccc}
\hline & 3 months & 6 months & 12 months \\
\hline Treatment-naïve $(\mathrm{n}=9)$ & & & \\
Gain of $\geq 5$ letters $n(\%)$ & $2(22.2)$ & $2(22.2)$ & $5(55.6)$ \\
Gain of $\geq 10$ letters $n(\%)$ & $1(11.1)$ & $1(11.1)$ & $3(33.3)$ \\
Gain of $\geq 15$ letters $n(\%)$ & $0(0.0)$ & $0(0.0$ & $1(11.1)$ \\
Loss of $\geq 5$ letters $n(\%)$ & $3(33.3)$ & $2(22.2)$ & $2(22.2)$ \\
Loss of $\geq 10$ letters $n(\%)$ & $1(11.1)$ & $1(11.1)$ & $0(0.0)$ \\
Loss of $\geq 15$ letters $n(\%)$ & $0(0.0)$ & $0(0.0)$ & $0 .(0.0)$ \\
Mean change in CMT $(\mu \mathrm{m} \pm \mathrm{SD})$ & $161 \pm 185$ & $188 \pm 161$ & $172 \pm 164$ \\
& & & \\
Prior treatment $(\mathrm{n}=42)$ & $22(52.4)$ & $28(66.7)$ & $31(73.8)$ \\
Gain of $\geq 5$ letters $n(\%)$ & $13(31.0)$ & $18(42.9)$ & $19(45.2)$ \\
Gain of $\geq 10$ letters $n(\%)$ & $7(16.7)$ & $11(26.2)$ & $8.0(19)$ \\
Gain of $\geq 15$ letters $n(\%)$ & $4(9.5)$ & $5(11.9)$ & $2(4.8)$ \\
Loss of $\geq 5$ letters $n(\%)$ & $0(0.0)$ & $3(7.1)$ & $1(2.4)$ \\
Loss of $\geq 10$ letters $n(\%)$ & $0(0.0)$ & $2(4.8)$ & $1(2.4)$ \\
Loss of $\geq 15$ letters $n(\%)$ & $164 \pm 131$ & $220 \pm 162$ & $205 \pm 189$ \\
Mean change in CMT $(\mu \mathrm{m} \pm \mathrm{SD})$ & \\
\hline
\end{tabular}

The effect of treatment on VA and CMT for the $n=42$ eyes that had received treatment for diabetic retinopathy or maculopathy (macular laser, pan-retinal photocoagulation, intravitreal Avastin, or Triamcinolone) prior to intravitreal ranibizumab.
The data were analysed by subgroups of eyes by their baseline VA (baseline VA $<55$ letters and baseline $\mathrm{VA} \geq 55$ letters) as well as the baseline CMT (baseline $\mathrm{CMT}<500$ and baseline CMT $\geq 500$ ). Table 3 shows the baseline and change in VA and CMT for the eyes split into these groups. Figure 1 is a comparison of baseline VA and the change in VA (number of letters) over the 12-month period for all eyes. Subjects with a baseline VA less than 55 letters are depicted on the left-side of the figure.

The ranibizumab injections were tolerated well by all subjects. There were no procedure-related serious adverse events and no reported adverse events. Two subjects suffered myocardial infarction. One patient with a history of hypertension and ischaemic heart disease suffered myocardial infarction 4 months following the first and only injection, and was excluded from the study and these results. The other, with a history of stroke, transient ischaemic attack, and hypertension suffered fatal myocardial infarction 2 months after the end of the study. Neither of these adverse events is thought to be related to the anti-vegf treatment.

\section{Discussion}

Several pivotal studies have demonstrated greater efficacy of intravitreal ranibizumab injections for the treatment of $\mathrm{DMO}^{2,3,5,13}$ compared with sham injections ${ }^{2,4}$ or laser. ${ }^{3,13}$ None of these studies had sizeable South Asian populations. The RISE and RIDE studies had only $3.1 \%$ and $5.4 \%$ of patients from Asian backgrounds, respectively. ${ }^{4}$ The majority of their subjects (78.2 and $80.6 \%$ ) are reported to be White. The READ2 study also reports a predominantly Caucasian subject group (76\% for group 1), which received ranibizumab injections only. ${ }^{3}$

Ethnicity is known to influence the prevalence of diabetes, albeit in a complex manner, with many overlapping co-factors. ${ }^{1,7-11}$ In the UK, the prevalence of diabetes within a population of South Asian background is higher than the general population. ${ }^{10,11}$ Locally, $74.9 \%$ of the NHS Bradford City clinical commissioning group

Table 3 Comparison of age, visual acuity, and CMT data for subjects with poorer baseline VA ( $<55$ letters) and better baseline VA $(\geq 55$ letters) and for subjects with thinner $(<500 \mu \mathrm{m})$ and thicker $(\geq 500 \mu \mathrm{m})$ baseline CMT

\begin{tabular}{lcccc}
\hline & $\begin{array}{c}\text { Baseline } V A<55 \\
\text { letters }\end{array}$ & $\begin{array}{c}\text { Baseline VA } \geq 55 \\
\text { letters }\end{array}$ & $\begin{array}{c}\text { Baseline } \\
\text { CMT }<500 \mu m\end{array}$ & $\begin{array}{c}\text { Baseline } \\
C M T \geq 500 \mu m\end{array}$ \\
\hline$N$ & 29 & 36 & 23 & 28 \\
Mean age (years \pm SD) & $65 \pm 11$ & $61 \pm 11$ & $65 \pm 12$ & $61 \pm 11$ \\
Mean VA (letters \pm SD) & $43.8 \pm 8.2$ & $66.4 \pm 5.9$ & $55.2 \pm 13.4$ & $53.8 \pm 12.7$ \\
Mean change VA to 6 months (letters \pm SD) & $8.9 \pm 8.8$ & $3.7 \pm 11.0$ & $8.4 \pm 7.4$ & $3.8 \pm 12.4$ \\
Mean change VA to 12 months (letters \pm SD) & $10.5 \pm 12.1$ & $6.6 \pm 7.2$ & $10.5 \pm 10.6$ & $6.9 \pm 9.3$ \\
Mean change CMT to 6 months $(\mu \mathrm{m} \pm$ SD) & $195 \pm 190$ & $215 \pm 112$ & $156 \pm 96$ & $253 \pm 187$ \\
Mean change CMT to 12 months $(\mu \mathrm{m} \pm$ SD) & $241 \pm 219$ & $159 \pm 134$ & $145 \pm 143$ & $244 \pm 203$ \\
\hline
\end{tabular}


(CCG) population is South Asian, ${ }^{14}$ where prevalence of known diabetes is $8.9 \%$ compared with $6.7 \%$ in similar sized CCGs. ${ }^{15}$ The prevalence of diabetes can be $2.5-5$ times more common among South Asians than the general population. ${ }^{10,11}$ In addition, DRIVE UK ${ }^{1}$ reported that sight-threatening diabetic retinopathy was significantly more prevalent in Afro-Caribbeans (11.5\%) and South Asians (10.3\%) compared with white Europeans (5.5\%). DMO is more prevalent amongst diabetics with an Asian background than Caucasians. ${ }^{1}$

Table 4 shows a comparison of mean VA gain, mean CMT change, and average number of injections at 6 and 12 months compared with other ranibizumab studies over 6, 12, and 24 months as stated. ${ }^{2,4,13}$ The mean VA improvement in ADMOR was 8.5 letters at 12 months compared with 6.1 letters in the RESTORE ${ }^{13}$ study, and 10.3 letters in RESOLVE. ${ }^{2}$ Ranibizumab and deferred laser group at 1 year reported 8.6 letters gain in DRCRnet protocol I study. ${ }^{16}$ The mean baseline VAs of READ2, RESOLVE, and ADMOR are similar, ranging between 55.7 and 60.2 letters. ${ }^{2,3,13}$ The important differences between these studies and ADMOR is that ADMOR has a

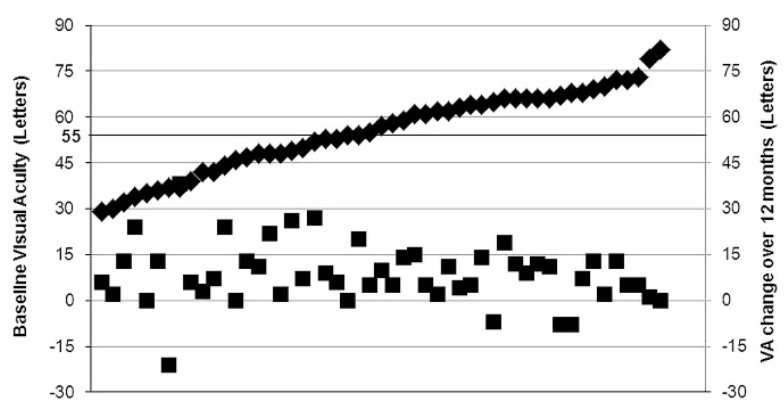

Figure 1 A comparison of baseline VA and the change in VA (number of letters) over the 12-month period for all 51 eyes. Filled squares $=$ baseline VA (letters), filled diamonds $=$ VA change over 12 months. higher mean baseline HbA1c. Furthermore, most eyes in ADMOR had previous treatments, which is a surrogate marker for longer duration of DMO.

None of the subjects in this study had recent onset DMO ( $<6$ months), with only 9 of the 51 eyes having had no prior treatment (macular laser, intravitreal bevacizumab, intravitreal Triamcinolone, or pan-retinal photocoagulation). The average number of prior treatments per eye was two (maximum seven treatments) and the mean improvement in VA was 8.5 letters at 12 months. This is also comparable with READ2 where laser-treated patients were switched to ranibizumab in year $2 .{ }^{3}$ Interestingly of the 31 eyes with two or more prior interventions, 11 (35\%) achieved $\geq 15$ letters improvement by 12 months. Paradoxically, none of the 26 eyes with less than two prior interventions, gained $\geq 15$ letters improvement. When considering the CMT, the mean difference between baseline and 12 months was similar for the two groups (pre-treated group: $205 \mu \mathrm{m}$, naïve group: $173 \mu \mathrm{m}$ ). In agreement with other studies, ${ }^{17-19}$ this finding points to the complex relationship between VA and CMT, implying that simply reducing oedema does not guarantee improvement in visual function. Concurring with DRCRnet study, results from ADMOR imply that patients who had received previous treatments for DMO can still benefit from ranibizumab injections. Conversely, patients with relatively recent DMO may still respond poorly to treatment. Further investigation with greater numbers of subjects is required to investigate the precise cause (or causes) for nonresponders or poor responders.

As can be seen from Table 4, VA gains of 6.1 to 12 letters for 12-24-month periods have been reported. ${ }^{2,3,4,13}$ It was reported that studies with fixed dosing 4,5 had better visual outcomes than studies with prn or extended treatment intervals. ${ }^{2,13}$ However, the recent DRCRNet protocol T study ${ }^{6}$ has reported very good results with as

Table 4 The mean change in VA and CMT as well as the average number of injections for the current study at 6 and 12 months

\begin{tabular}{|c|c|c|c|c|}
\hline & $\begin{array}{c}\text { Mean change in } \\
V A \text { (no. of letters) }\end{array}$ & $\begin{array}{l}\text { Mean change } \\
\text { in CMT }(\mu \mathrm{m})\end{array}$ & $\begin{array}{c}\text { Average } \\
\text { no. of injections }\end{array}$ & Notes \\
\hline \multirow[t]{2}{*}{ ADMOR } & $5.8 \pm 10.3$ & $214 \pm 161$ & $5.2 \pm 1.2$ & 6-month data \\
\hline & $8.5 \pm 10.0$ & $199 \pm 184$ & $7.4 \pm 2.3$ & 12-month data \\
\hline DRCRnet I prompt laser ${ }^{a}$ & 7.5 & $142(375)$ & 8 (median) & 68 weeks \\
\hline DRCRnet I Deferred Laser ${ }^{a}$ & 8.6 & $132(395)$ & 9 (median) & 68 weeks \\
\hline \multirow[t]{2}{*}{ READ2 (group 1) } & 7.24 & 106.3 & 5.3 & 6-month data \\
\hline & 7.70 & & 9.3 & 24-month data \\
\hline RESTORE & $6.1 \pm 6.43$ & $118.7 \pm 115.07$ & $\mathrm{n} / \mathrm{a}$ & 12-month data \\
\hline RESOLVE & $10.3 \pm 9.1$ & $194.2 \pm 135.1$ & $10.2 \pm 2.5$ & 12-month data \\
\hline RISE & $11.9 \pm 12.1$ & $253.1 \pm 183.7$ & 24 & 24-month data \\
\hline RIDE & $12.0 \pm 14.9$ & $270.7 \pm 201.6$ & 24 & 24-month data \\
\hline
\end{tabular}

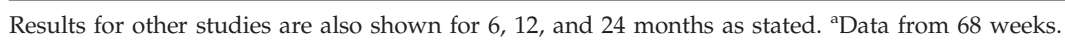


required treatment where close monthly follow-up can be assured. There is potential for eyes to gain more vision beyond 12 months (Table 4) when fixed monthly dosing was followed. ${ }^{4}$ A longer period of follow-up on the ADMOR cohort can help establish if this is also true in South Asians.

All pivotal studies showed a reduction in CMT with ranibizumab injections, however, the mean changes in CMT varied. ${ }^{2-5,13}$ The mean reduction in CMT at 12 months in ADMOR was $199 \pm 184 \mu \mathrm{m}$. As OCT thickness varies with different OCT machines, direct comparison of ADMOR CMT findings with other studies needs to be interpreted with care. Nonetheless, the DRCRnet protocol I reported a mean reduction of $169 \mu \mathrm{m}$ at 1 year in ranibizumab-deferred laser group. ${ }^{16}$

The 12- and 24-month mean changes in CMT for the other studies vary greatly (118.7-270.7 $\mu \mathrm{m}$, Table 4), and some of this difference can be explained by patient selection. In the current study, because of NICE guidelines, all eligible eyes had a baseline CMT of $>400 \mu \mathrm{m}$. Other studies have either not specified a minimum CMT in their inclusion criteria $^{2,13}$ or specified minimum CMT of $\geq 250^{3}$ or $\geq 275 \mu \mathrm{m} .{ }^{4}$ If the mean baseline CMT was lower, as is the case with all these studies, it would be expected that the change in CMT over time would be less owing to a floor effect. Comparing the 6-month data, the READ2 study ${ }^{3}$ required a central subfield thickness of $\geq 250 \mu \mathrm{m}$ compared with $>400 \mu \mathrm{m}$ in this study. As expected, the mean baseline CMT in READ2 $(420.1 \mu \mathrm{m})$ was lower than ADMOR $(532 \pm 129 \mu \mathrm{m})$, thus potentially limiting the reduction in CMT, particularly as $250 \mu \mathrm{m}$ is considered to be within the normal range for CMT using a spectral domain OCT. ${ }^{17}$ The 12-month data for the RESTORE study also showed much less reduction in CMT compared with ADMOR, and similarly the baseline CMT was less $(426.6 \pm 118.01 \mu \mathrm{m})$.

At both 6 and 12 months, ADMOR results show a consistent trend for eyes with poorer baseline VA to have better visual gain compared with eyes with a better baseline VA. However, there is no consistent difference with the change in CMT between the two groups over these two time periods. Although greater numbers are required for statistical analysis of this heterogeneous group, these findings agree with other studies that have investigated the relationship between OCT-measured CMT and VA in DMO. ${ }^{17-19}$ Results show a weak to modest correlation, with at times unpredictable reduction of VA with decrease in CMT. ${ }^{18}$ Better understanding of the anatomical changes depicted on OCT is required to specifically assess neuronal survival, which can then be better correlated with VA. ${ }^{19}$

Looking at the baseline structure as a predictor for outcome, ADMOR found that the mean baseline VA in eyes with a thinner CMT $(55.2 \pm 13.4)$ was not significantly different to the baseline VA of eyes with a thicker CMT $(53.8 \pm 12.7)$ (unpaired $t$-test $P=0.07)$. The group of eyes with thinner baseline CMT achieved a greater mean improvement in VA at 12 months, than those with thicker baseline CMT, however, the reduction in CMT was less. In general, both groups showed a mean improvement in VA following ranibizumab treatment, yet despite a greater reduction in CMT, those subjects with initially worse oedema, showed less VA improvement. (Table 3) Intuitively, thinner the baseline CMT, the earlier the disease state and hence less permanent structural damage and therefore greater the potential for improvement. This trend would favour treating DMO early rather than later.

In the ADMOR cohort, only one eye lost more than two lines of acuity over the course of the study. This eye developed progressive ischaemic maculopathy that was diagnosed at his 12-month appointment. This patient had received prior macular laser and had poor diabetic control throughout the study (HbA1c 84). These factors can adversely influence the efficacy of any intervention.

Although some comparisons can be made between ADMOR and other studies, there are some limitations to this study. ADMOR has small patient numbers, based at a single clinic, with follow-up limited to 12 months, and it is not a randomised controlled trial. However, all consecutive South Asian subjects were selected and they received ranibizumab injections as per the DRCRnet Protocol I. As such, the data provide real-life experience of the effect of ranibizumab on DMO in South Asian eyes. South Asian diabetic patients were selected for this study in order to understand how this particular group of patients responds to ranibizumab in comparison with the (usually mostly Caucasian) populations cited in the literature.

\section{Conclusions}

Ranibizumab $0.5 \mathrm{mg}$ is safe and effective at reversing vision loss due to DMO in patients of South Asian origin at 12 months. Ranibizumab appears to have similar efficiency in South Asian patients as reported in the Caucasians. Ranibizumab is effective in eyes with longstanding DMO that were treated previously with laser. Visual and anatomical outcomes similar to previous studies can be achieved in routine clinical practice for treatment of naïve as well as previously treated DMO with ranibizumab. Further studies are needed to define long-term outcome in patients of different ethnicity and DMO. 


\section{Summary}

\section{What was known before}

- The prevalence of diabetes as well as the severity of diabetic retinopathy is higher in South Asians compared with Caucasians. Furthermore, diabetics of South Asian origin are twice as likely to develop DMO as Caucasians.

- Ranibizumab has been shown to be effective in treatment of DMO in studies based on mainly Caucasian populations. However, there are little useful data on South Asians.

\section{What this study adds}

- This paper describes the first cohort of South Asian patients with DMO who underwent ranibizumab treatment for DMO and completed 12 months follow-up.

- Ranibizumab $0.5 \mathrm{mg}$ is safe and effective at reversing vision loss due to DMO in patients of South Asian origin at 12 months.

- Ranibizumab treatment appears to be effective in Asian patients with long-standing DMO who received prior laser treatments.

\section{Conflict of interest}

FG has received educational grants from Novartis, Bayer, and carried out consultancy for Novartis, Bayer, Allergan, and Alimera. FG and $\mathrm{CH}$ are involved in research studies by Novartis, Bayer. FG has received educational grants from Novartis. The department receives research funding from Novartis.

\section{Acknowledgements}

We would like to thank consultant ophthalmologists Tomas Cudrnak, Helen Devonport, and Roopa Setty, who worked at the Macula Clinic during the course of the study.

\section{References}

1 Sivaprasad S, Gupta B, Gulliford MC, Dodhia H, Mann S, Nagi $\mathrm{D}$ et al. Ethnic variations in the prevalence of diabetic retinopathy in people with diabetes attending screening in the United Kingdom (DRIVE UK). PLoS One 2012; 7: e32182.

2 Massin P, Bandello F, Garweg JG, Hansen L, Larsen M, Mitchell $\mathrm{P}$ et al. Safety and efficacy of ranibizumab in diabetic macular edema (RESOLVE Study). Diabetes Care 2010; 33: 2399-2405.

3 Nguyen QD, Shah SM, Khwaja AA, Channa R, Sepah YJ, Sophie $\mathrm{R}$ et al. Two-year outcomes of the ranibizumab for edema of the macula in Diabetes (READ-2) Study. Ophthalmology 2010; 117: 2146-2151.

4 Nguyen QD, Brown DM, Marcus DM, Boyer DS, Patel S, Feiner $L$ et al. Ranibizumab for diabetic macular edema. Results from 2 Phase III Randomized trials: RISE and RIDE. Ophthalmology 2012; 119: 789-801.
5 Brown DM, Nguyen QD, Marcus DM, Boyer DS, Patel S, Feiner $\mathrm{L}$ et al. Long-term outcomes of ranibizumab therapy for diabetic macular edema: The 36-month results from two phase III Trials. RISE and RIDE. Ophthalmology 2013; 120: 2013-2022.

6 Wells JA, Glassman AR, Ayala AR, Jampol LM, Aiello LP, Antoszyk AN et al. Diabetic Retinopathy Clinical Research Network Aflibercept, Bevacizumab, or Ranibizumab for Diabetic Macular Edema. N Engl J Med 2015; 372(13): 1193-1203.

7 Sivaprasad S, Gupta B, Crosby-Nwaobi R, Evans J. Prevalence of diabetic retinopathy in various ethnic groups: A world-wide perspective. Surv Ophthalmol 2012; 57: 347-370.

8 Gupta B. http://dspace.lib.cranfield.ac.uk/handle/ 1826/7165 (accessed 13 May 2014).

9 Diabetes UK, Diabetes in the UK 2012. Key statistics on diabetes. Available from http://www.diabetes.org.uk/ Documents/Reports/Diabetes-in-the-UK-2012.pdf (accessed 14 January 2014).

10 Health Survey for England 2004. Health of ethnic minorities. Available from www.ic.nhs.uk/pubs/hse04ethnic (accessed 14 Jan 2014).

11 Dreyer G, Hull S, Aitken Z, Chesser A, Yaqoob MM. The effect of ethnicity on the prevalence of diabetes and associated chronic kidney disease. QJM 2009; 102: 261-269.

12 Diabetic Retinopathy Clinical Research Network, Aiello LP, Beck RW, Bressler NM, Browning DJ, Chalam KV, Davis M et al. Rationale for the diabetic retinopathy clinical research network treatment protocol for centre-involving diabetic macular edema. Ophthalmology 2011; 118: e5-e14.

13 Mitchell P, Bandello F, Schmidt-Erfurth U, Lang GE, Massin P, Schlingemann RO et al. The RESTORE Study. Ranibizumamb monotherapy or combined with laser versus laser monotherapy for diabetic macular edema. Ophthalmology 2011; 118: 615-625.

14 Bradford City CCG. Available from http://www. bradfordcityccg.nhs.uk/wp-content/uploads/2013/10/ Bradford-Citys-objectives-for-website.pdf (accessed 13 May 2015).

15 Diabetes Community Health Profile for Bradford and Airedale PCT. http://www.yhpho.org.uk/ diabetescommunityhealthprofiles/default.aspx.

16 Elman MJ, Qin H, Aiello LP, Beck RW, Bressler NM, Ferris FL III et al. Intravitreal ranibizumab for diabetic macular edema with prompt vs deferred laser treatment: 3-year randomized trial results. Ophthalmology 2012; 119: 2312-2318.

17 Leung CK, Cheung CY, Weinreb RN, Lee G, Lin D, Pang CP et al. Comparison of macular thickness measurements between time domain and spectral domain optical coherence tomography. Invest Ophthalmol Vis Sci 2008; 49: 4893-4897.

18 Alasil T, Pearse KA, Updyke JF, Dustin L, Ouyang Y, Walsh AC et al. Relationship between optical coherence tomography retinal parameters and visual acuity in diabetic macular edema. Ophthalmology 2010; 117: 2379-2386.

19 Pelosini L, Hull CC, Boyce JF, McHugh D, Stanford MR, Marshall J. Optical coherence tomography may be used to predict visual acuity in patients with macular edema. Invest Ophthalmol Vis Sci 2011; 52: 2741-2748. 